PROCEEDINGS OF THE

AMERICAN MATHEMATICAL SOCIETY

Volume 130, Number 5, Pages 1457-1458

S 0002-9939(01)06522-4

Article electronically published on December 27, 2001

\title{
PARALLEL TANGENT HYPERPLANES
}

\author{
SOL SCHWARTZMAN
}

(Communicated by Ralph Cohen)

\begin{abstract}
Let $\Sigma^{2 n}$ be a smooth strictly convex closed hypersurface in $R^{2 n+1}$ and let $M^{2 n}$ be any oriented smooth connected manifold immersed in $R^{2 n+1}$. Suppose that $f$ is a continuous function from $\Sigma^{2 n}$ to $M^{2 n}$. Then there is at least one point $p \in \Sigma^{2 n}$ such that the hyperplane tangent to $\Sigma^{2 n}$ at $p$ is parallel to the hyperplane tangent to the immersed manifold $M^{2 n}$ at the point corresponding to $f(p)$. If there did not exist at least two such points, $M^{2 n}$ would have to be compact and the Hurewicz homomorphism of $\pi_{2 n}\left(M^{2 n}\right)$ into $\mathrm{H}_{2 n}\left(M^{2 n}\right)$ would have to be surjective. If in addition our immersion was an embedding, the Euler characteristic of $M^{2 n}$ would have to be equal to \pm 2 . For any $\Sigma^{2 n}$ and any immersed $M^{2 n}$ we could always get maps $f$ for which the number of points $p$ satisfying the conditions of our theorem exactly equaled two. An example can be given in which both $\Sigma^{2 n}$ and $M^{2 n}$ are the unit sphere about the origin in $R^{2 n+1}$ and there is only one such point $p$.
\end{abstract}

Let $\Sigma^{2 n}$ be a smooth strictly convex closed hypersurface of class $C^{2}$ in $R^{2 n+1}$. $\Sigma^{2 n}$ is said to be strictly convex provided the second fundamental form on $\Sigma^{2 n}$ is everywhere positive definite. Let $M^{2 n}$ be any oriented connected manifold of class $C^{1}$ and let $i$ be an immersion of $M^{2 n}$ into $R^{2 n+1}$ of class $C^{1}$. Let $f$ be a continuous function from $\Sigma^{2 n}$ to $M^{2 n}$.

Theorem 1. There exists at least one point $p \in \Sigma^{2 n}$ such that the tangent hyperplane to $\Sigma^{2 n}$ at $p$ is parallel to the tangent hyperplane to $i\left(M^{2 n}\right)$ at $i(f(p))$.

Proof. Let $G_{1}$ and $G_{2}$ respectively be the Gauss maps from $\Sigma^{2 n}$ and $M^{2 n}$ to the unit sphere in $R^{2 n+1}$. It is well known that $G_{1}$ must be an orientation preserving homeomorphism. Let $F$ denote the map $G_{1}^{-1} \circ G_{2} \circ f$ from $\Sigma^{2 n}$ to itself, and let $F_{\star}$ be the map of the one-dimensional space $\mathrm{H}_{2 n}\left(\Sigma^{2 n}, R\right)$ into itself induced by $F$. If the trace of $F_{\star}$ is different from -1 , the Lefschetz number of the map $F$ will be different from zero so we can be sure that $F$ has a fixed point $p$. However saying that $p$ is a fixed point of $F$ just says that the unit normal to $\Sigma^{2 n}$ at $p$ is identical to the unit normal to $i\left(M^{2 n}\right)$ at $i(f(p))$. Similarly if $D$ is the antipodal map of the unit sphere in $R^{2 n+1}$ onto itself and we let $\bar{F}$ be the map $G_{1}^{-1} \circ D \circ G_{2} \circ f$ of $\Sigma^{2 n}$ into itself, saying that $p$ is a fixed point of $\bar{F}$ just says that the unit normal to $\Sigma^{2 n}$ at $p$ is the negative of the unit normal to $i\left(M^{2 n}\right)$ at $i(f(p))$. Thus to prove our theorem we need only show that either the Lefschetz number of $F$ is different from zero or the Lefschetz number of $\bar{F}$ is different from zero.

Received by the editors August 1, 2000.

2000 Mathematics Subject Classification. Primary 53A07, 55M20.

(C)2001 American Mathematical Society 
However the only way that the map $F$ can have Lefschetz number zero is if $F_{\star}$ has trace -1 and the only way that $\bar{F}$ can have Lefschetz number zero is if $F_{\star}$ has trace 1 . Thus our theorem is proved.

Theorem 2. Suppose that the hypotheses of Theorem 1 hold and there is only one point $p$ such that the conclusion of Theorem 1 holds. Then $M^{2 n}$ must be compact and the Hurewicz map of $\pi_{2 n}\left(M^{2 n}\right)$ to $\mathrm{H}_{2 n}\left(M^{2 n}, Z\right)$ is surjective. If in addition our immersion $i$ is an embedding, then the Euler characteristic of $M^{2 n}$ must equal \pm 2 .

Proof. By our previous argument if there is only one point $p$ such that the conclusion of Theorem 1 holds, then the trace of $F_{\star}$ must equal either 1 or -1 . This would just say that the map $F_{\star \star}$ of $\mathrm{H}_{2 n}\left(\Sigma^{2 n}, Z\right)$ to itself induced by $F$ is an isomorphism. For this to hold both the map $f_{\star}$ of $\mathrm{H}_{2 n}\left(\Sigma^{2 n}, Z\right)$ to $\mathrm{H}_{2 n}\left(M^{2 n}, Z\right)$ induced by $f$ and the map $\left(G_{2}\right)_{\star}$ of $\mathrm{H}_{2 n}\left(M^{2 n}, Z\right)$ to the corresponding homology group of the unit sphere in $R^{2 n+1}$ must be isomorphisms. Therefore, if $F_{\star \star}$ is an isomorphism, that implies that $M^{2 n}$ is compact and that the Hurewicz map of $\pi_{2 n}\left(M^{2 n}\right)$ to $\mathrm{H}_{2 n}\left(M^{2 n}, Z\right)$ must be surjective. Since $G_{2}$ is the Gauss map associated with the immersed manifold $M^{2 n}$, saying $\left(G_{2}\right)_{\star}$ is an isomorphism just says that the degree of the Gauss map is \pm 1 . However if $i$ is an embedding it is known that the degree of the Gauss map is one half the Euler characteristic. Thus the Euler characteristic of $M^{2 n}$ must be 2 or -2 .

Now suppose that $\Sigma^{2 n}, M^{2 n}$ and $i$ are given. Then it is easy to see that for any constant map $f$ of $\Sigma^{2 n}$ into $M^{2 n}$ there are exactly two points satisfying the conditions stated in the conclusion of Theorem 1 .

Finally let $\Sigma^{2 n}$ and $M^{2 n}$ both be the unit sphere about the origin in $R^{2 n+1}$. It is well known that there exists a smooth vector field on $\Sigma^{2 n}$ which vanishes at just one point and which is such that the associated flow has no nonstationary periodic orbits. If we let points on $\Sigma^{2 n}$ move for a sufficiently short time under the action of this flow, the corresponding diffeomorphism will have exactly one fixed point and no point will be sent into the diametrically opposite point. It follows that for this diffeomorphism of $\Sigma^{2 n}$ onto $M^{2 n}=\Sigma^{2 n}$ there is exactly one point $p$ such that the hyperplane tangent to $\Sigma^{2 n}$ at $p$ is parallel to the hyperplane tangent to $M^{2 n}$ at the image of $p$.

It is known that an orientable surface other than a sphere is a $K(\pi, 1)$ space, so the Hurewicz homomorphism in dimension two cannot be an isomorphism. Consequently when $n=1$ the only case in which there can be only one point $p$ is when $M^{2}$ is a topological sphere.

Department of Mathematics, University of Rhode Island, Kingston, Rhode Island 02881 